\title{
Applicability of Bardeen-Cooper-Schrieffer theory to small-sized superconductors: role of Cooper-pair binding energy
}

\author{
W. V. Pogosov ${ }^{1,2, *}$ \\ (1) Center for Fundamental and Applied Research, \\ N. L. Dukhov All-Russia Research Institute of Automatics, Sushchevskaya 22, 127055 Moscow, Russia \\ (2) Institute for Theoretical and Applied Electrodynamics, \\ Russian Academy of Sciences, Izhorskaya 13, 125412, Moscow, Russia and \\ (*) Corresponding author. Tel. +7-495-3625147; Fax+7-495-4842633; e-mail: Walter.Pogosov@gmail.com
}

\begin{abstract}
We analyze conditions of applicability of grand-canonical mean-field Bardeen-Cooper-Schrieffer theory to the evaluation of an interaction energy in the ground state of small-sized superconductors. We argue that this theory fails to describe correctly an interaction energy, when an average distance between energy levels near the Fermi energy due to the size quantization becomes of the order of the single-pair binding energy. In conventional superconductors, this quantity is much smaller than the superconducting gap.
\end{abstract}

PACS numbers: $74.20 . \mathrm{Fg}, 03.75 . \mathrm{Hh}$

\section{INTRODUCTION}

It is known that Cooper pairing between electrons in conventional superconductors is due to their interaction through phonons. Basic properties of such superconductors can be described within the framework of BardeenCooper-Schrieffer (BCS) theory of superconductivity. In the BCS theory $\underline{\underline{1}}$, electron pairing is accounted for by the interaction term in the Hamiltonian, which couples only electrons with up and down spins on the same energy level (reduced BCS Hamiltonian).

Furthermore, the problem is solved approximately by using a mean-field approach. There are two well-known realizations of this approach in connection to the BCS theory. The first one uses a variational ansatz for the many-body wave function of the electron system 1 . The second one is based on the Bogoliubov canonical transformation 2 . It turns out that both methods give essentially the same results. In addition, in both approaches a grand-canonical description (electron number is not fixed) is encoded which becomes asymptotically exact in the macroscopic limit.

Thus, BCS theory contains two sources of inaccuracies, namely, mean-field method to solve the many-body problem and the grand-canonical description. It was shown that inaccuracies of the first type become negligible in a macroscopic limit due to the very peculiar form of the interaction term of the reduced BCS Hamiltonian ${ }^{3-5}$ (however, see a recent study of correlation functions in Ref ${ }^{6}$ ). Hence, mean-field BCS results for the low-lying part of energy spectrum, including an important expression for the superconducting gap, are asymptotically exact in the thermodynamical limit (corrections are proportional to the inverse number of particles). Note that there also exist formulations of BCS theory with fixed number of particles ${ }^{?}$.

However, when the system size is decreasing, one can expect that macroscopic BCS results at certain stage become no longer applicable, since inaccuracies of both types start to be significant (despite of the fact that the reduced BCS Hamiltonian itself is adequate for the case of metallic grains $\left.{ }^{8}\right)$.

A problem of applicability of the mean-field BCS treatment to small-sized systems was addressed in literature for decades. A widely spread point of view is due to the well-known paper of Matveev and Larkin ${ }^{9}$, who concentrated on parity effects. They introduced a parameter aimed to characterize these effects, this quantity being defined as $\Delta_{M L}=E_{2 l+1}-\frac{1}{2}\left(E_{2 l}+E_{2 l+2}\right)$, where $E_{N}$ is a ground state energy as a function of the number of electrons. In the macroscopic limit, $\Delta_{M L}$ reduces to the usual superconducting gap $\Delta$. Matveev and Larkin have shown, by considering deviations from the meanfield treatment that $\Delta_{M L}$ starts to differ from $\Delta$, provided that a typical distance between one-electron energy levels $\delta \varepsilon$ due to the size quantization becomes of the order of $\Delta$. From this result it is usually concluded that BCS theory is adequate until $\delta \varepsilon<\Delta$. Indeed, $\Delta$ yields a natural energy scale associated with the Cooper pairing and, therefore, such a conclusion is expectable.

A parity effect, as described by the Matveev-Larkin parameter $\Delta_{M L}$, is an important characteristic of the interaction in the Cooper channel. However, it is definitely not the only one quantity which is fully determined by it. Another quite natural quantity is an interaction energy in the ground state, when all electrons are paired, as well as a closely related quantity, which is a condensation energy. The interaction energy is an energy difference between the ground states of the same electron system without and with the interaction. For the condensation energy we use a definition of Ref $\underline{\underline{10}}$. Namely, it is the difference between the expectation value of BCS Hamiltonian in the Fermi ground state and the exact ground state energy. It is more difficult to probe experimentally the ground state, but the condensation energy influences, for example, spin magnetization of grains ${ }^{10}$.

The evolution of the ground state energy upon changing system's size was studied in Ref $\underline{10}$ within the conserved particle number approach and using the Richard- 
son exact solution of the reduced BCS Hamiltonian 11 . Quite surprisingly, it was shown in Ref $\underline{10}$ that the condensation energy starts to deviate from the prediction of the grand-canonical BCS theory much earlier compared to what could be expected from the Matveev-Larkin criterion (see also Ref ${ }^{12}$ ). Namely, it happens when $\delta \varepsilon$ becomes of the order of $\Delta^{2} / \omega_{D}$, where $\omega_{D}$ is a Debye frequency. Since in conventional superconductors $\Delta \ll \omega_{D}$, this quantity is much smaller than $\Delta$. Let us stress that, of course, this result does not contradict a general conclusion on the asymptotical accuracy of the mean-field BCS solution in the thermodynamical limit. Nevertheless, the result of Ref $\frac{10}{\underline{1}}$ seems to be rather puzzling, since it is believed that there is only a single energy scale characterizing superconducting correlations which is a gap $\Delta$. It implies that there is also another scale $\Delta^{2} / \omega_{D}$, but the physical meaning of this quantity as well as its origin are not so clear.

In this paper, we present various arguments in favor of the point of view that the additional energy scale reported in Ref $\underline{10}$ is nothing but a binding energy of a single pair found by Cooper long time ago $\frac{13}{2}$. We point out that the criterion of Ref $\underline{10}$ is in an agreement with the approximate expression for the ground state energy obtained recently in Ref $\stackrel{14}{=}$, where single-pair binding energy was used as a 'building block' for the total energy of the many-pair system. We also demonstrate that a criterion involving $\Delta^{2} / \omega_{D}$ can be recovered using a perturbation theory or simple dimensional estimates. A concept of an energy scale given by a binding energy of a single Cooper pair was already introduced in Refs $\underline{16,17}$ in connection to the macroscopic BCS limit. Single-pair binding energy provides a hidden energy scale which was used to develop a novel interpretation of known BCS results 16 . Arguments of this paper suggest that this energy scale becomes explicit in small-sized systems, where deviations from the mean-field treatment are significant.

\section{MODEL}

We consider a system of finite sizes which contains electrons with up and down spins. This system is described by the reduced BCS Hamiltonian, $H=H_{0}+\mathcal{V}$. The first term, $H_{0}$, is a kinetic energy

$$
H_{0}=\sum_{n} \varepsilon_{n}\left(a_{n \uparrow}^{\dagger} a_{n \uparrow}+a_{n \downarrow}^{\dagger} a_{n \downarrow}\right),
$$

where $a_{n \uparrow}^{\dagger}\left(a_{n \downarrow}^{\dagger}\right)$ is a creation operator for an electron in a discrete state $n$ with up (down) spin. The second term, $\mathcal{V}$, is responsible for the interaction between electrons with up and down spins:

$$
\mathcal{V}=-V \sum_{n, n^{\prime}} a_{n \uparrow}^{\dagger} a_{n \downarrow}^{\dagger} a_{n^{\prime} \downarrow} a_{n^{\prime} \uparrow} .
$$

This interaction acts only for the states within the 'Debye window', $E_{F}-\omega_{D}<\varepsilon_{n}<E_{F}+\omega_{D}$. The average spacing between neighboring energy levels within this interval is $\delta \varepsilon$. We take into account finiteness of the system size only by a nonzero value of $\delta \varepsilon$ which can be compatible to internal parameters characterizing superconducting correlations (such as the gap). It is sufficient, for the purposes of the present paper, to employ a so-called equally-spaced model which assumes that energy levels are distributed equidistantly within the Debye window. The density of states $N(0)$ within this window is equal to $1 / \delta \varepsilon$. A dimensionless interaction constant $v \equiv N(0) V$ is a characteristic of a given material and it can be taken nearly independent on system size $(v$ is small for most conventional superconductors). Thus, with the decrease of the system size, $\delta \varepsilon$ increases, while $V$ decreases.

By using standard mean-field methods, it is possible to derive BCS expression of the interaction energy. This quantity is extensive and coincides with the condensation energy up to intensive terms. In the macroscopic limit, the expression reads as

$$
E_{\text {int }}^{(B C S)} \simeq \frac{1}{2} N(0) \Delta^{2}=\frac{1}{2} \frac{\Delta^{2}}{\delta \varepsilon},
$$

where $\Delta \simeq 2 \omega_{D} \exp (-1 / v)$. In this limit, $\delta \varepsilon$ is the smallest energy scale in the system.

\section{RICHARDSON SOLUTION AND ELECTRON-HOLE SYMMETRY}

Instead of using a mean-field approximation, one can solve the BCS reduced Hamiltonian through the Richardson exact approach, which assumes a fixed number of electrons. Within this approach the problem is reduced to the resolution of the system of nonlinear algebraic equations (Bethe equations) for the set of energy-like quantities which determine a Hamiltonian eigen-state. Unfortunately, very few controllable analytical solutions are known for these equations $\frac{18}{}$. None of them covers an important crossover regime between the two limits: $\delta \varepsilon \gg \Delta$ and $\delta \varepsilon \ll \Delta$, where deviations from BCS results are significant.

Quite recently, a simple universal formula for the ground state energy was proposed in Ref $\frac{14}{\underline{14}}$. The derivation of Ref $\stackrel{14}{1}$ is based on symmetry between electron pairs and hole pairs for the reduced BCS Hamiltonian in the case of the equally-spaced model. It also uses certain assumption on the structure of the solution which is satisfied in all limits solvable analytically. The expression of interaction energy can be written as

$$
E_{\text {int }}^{(e-h)}=\frac{1}{2} \frac{\omega_{D}}{\delta \varepsilon}\left(\varepsilon_{c}+V\right),
$$

where $\varepsilon_{c}$ is given by the solution of a single Richardson equation:

$$
\frac{1}{V}=\sum_{n=0}^{2 \omega_{D} / \delta \varepsilon} \frac{1}{2 n \delta \varepsilon+\varepsilon_{c}}
$$


where $2 \omega_{D} / \delta \varepsilon$ is the number of one-electron energy levels in the Debye window. The latter equation, which in general case must be solved numerically, can be rewritten in terms of digamma-function:

$$
\frac{2 \delta \varepsilon}{V}=\psi\left(\frac{2 \omega_{D}}{\delta \varepsilon}+\frac{\varepsilon_{c}}{2 \delta \varepsilon}\right)-\psi\left(\frac{\varepsilon_{c}}{2 \delta \varepsilon}\right) .
$$

It is easy to see that $\varepsilon_{c}$ is nothing but the binding energy of a single pair, as introduced long time ago by Cooper $\underline{13}$. He considered a macroscopic system, $\omega_{D} / \delta \varepsilon \rightarrow \infty$, which however accommodates only a single pair in the Debye window, while in the BCS configuration this window is half-filled and the number of electron pairs in it, $\omega_{D} / \delta \varepsilon$, is macroscopically large.

Eqs. (4) and (5) provide 14 asymptotically exact results in the solvable limits $\delta \varepsilon \gg \Delta$ and $\delta \varepsilon \ll \Delta$. For instance, in the macroscopic limit, $\omega_{D} / \delta \varepsilon \rightarrow \infty$, the summation in Eq. (5) can be replaced by the integration, yielding $\varepsilon_{c}^{(\text {macro })} \simeq 4 \omega_{D} \exp (-2 / v)$. By substituting this expression to Eq. (4), we recover Eq. (3). Moreover, the comparison with the results of the numerical solution of the whole set of Richardson equations revealed a good accuracy of this approach in the crossover region 14 .

Since Eqs. (4) and (5) provide a good approximation for the crossover regime and contain physically meaningful quantities, one can answer a question under what conditions Eq. (3) is no longer accurate. It is easy to see from Eqs. (5) and (6) that this happens when it is not possible anymore to replace summation by integration in Eq. (5). It follows directly from Eq. (6) that the replacement is not allowed when $\delta \varepsilon \gtrsim \varepsilon_{c}^{(\text {macro })}$. This criterion for the interaction energy differs significantly from the condition obtained in Ref. $\underline{\underline{9}}$ for $\Delta_{M L}$, because $\varepsilon_{c}^{(\text {macro })} \ll \Delta$ at $v$ small. Note that the expressions of $\varepsilon_{c}^{(\text {macro })}$ and $\Delta$ look similarly, but these quantities differ dramatically due to the difference in the exponents.

Let us now discuss a connection of the obtained result with the conclusion of Ref. $\stackrel{10}{ }$, where it was found that BCS approach for condensation energy fails at $\delta \varepsilon \sim$ $\Delta^{2} / \omega_{D}$. Condensation energy differs from the interaction energy only by a contribution $v \omega_{D}$ and therefore the criteria of applicability of BCS theory to both these quantities are expected to be quite similar. A direct substitution of the expression of $\Delta$ shows that $\Delta^{2} / \omega_{D}$ is nothing but $\varepsilon_{c}^{\text {(macro) }}$ in a full agreement with our findings.

\section{PERTURBATION THEORY}

A similar criterion for the applicability of BCS theory to interaction energy can be recovered without turning to the exact Richardson solution, but using a perturbation theory. In order to demonstrate it, let us split the interaction term $\mathcal{V}$ of the Hamiltonian into a sum of two terms. The first term, $V_{0}$, is chosen in such a way that BCS solution corresponds to the exact eigen-states of $H_{0}+V_{0}$, while the second term, $\mathcal{W}$, can be treated as a perturbation. Actually, such a splitting can be performed by linearizing interaction term $\mathcal{V}$ of Hamiltonian in deviations of products of two fermionic operators from their mean values. The expressions of $V_{0}$ and $\mathcal{W}$ read as

$$
V_{0}=\frac{\Delta^{2}}{V}-\Delta \sum_{n}\left(a_{n \uparrow}^{\dagger} a_{n \downarrow}^{\dagger}+a_{n \downarrow} a_{n \uparrow}\right),
$$

$$
\mathcal{W}=-V\left(\sum_{n} a_{n \uparrow}^{\dagger} a_{n \downarrow}^{\dagger}-\Delta / V\right)\left(\sum_{n^{\prime}} a_{n^{\prime} \uparrow} a_{n^{\prime} \downarrow}-\Delta / V\right),
$$

while $\Delta$ is given by the usual condition

$$
\Delta=V \sum_{n}\left\langle a_{n \uparrow}^{\dagger} a_{n \downarrow}^{\dagger}\right\rangle,
$$

which makes it evident that the grand-canonical description is utilized within this approach.

It is easy to see that the interaction energy associated with $H_{0}+V_{0}$ is given by Eq. (3). When deriving this expression, one has to replace summation by the integration in the gap equation as well as in the expression of the ground state energy. This cannot be done if $\delta \varepsilon \gtrsim \Delta$. Otherwise, finite-size corrections within the mean-field approximation become large. However, a perturbation $\mathcal{W}$ responsible for fluctuations leads to significant complications. The effect of this perturbation in the thermodynamical limit was recently discussed in Ref $\underline{\underline{15}}$. The same technique can be applied for the system with finite $\delta \varepsilon$. Using Eq. (69) of Ref $\stackrel{15}{=}$, we readily find a first-order correction in $\mathcal{W}$ to the interaction energy as $v \omega_{D}$. It is of the same sign as the dominant contribution, i.e., superconducting correlations are enhanced by taking into account corrections beyond the mean-field approximation.

We now compare the dominant contribution to the interaction energy given by Eq. (3) with the first-order correction in $\mathcal{W}$. We find that they become of the same order at

$$
\delta \varepsilon \sim \frac{\Delta^{2}}{\omega_{D}} \ln \frac{2 \omega_{D}}{\Delta} .
$$

This quantity is much smaller than $\Delta$. Moreover, in the view of the relation $\Delta \ll \omega_{D}$ at small $v$, we can omit a weak logarithmic factor in the right-hand side of Eq. (10). Hence, we again arrive to the same criterion. Note that the first order correction in $\mathcal{W}$ to the condensation energy vanishes. However, one can estimate a secondorder correction in $\mathcal{W}$ using Eq. (77) of Ref $\frac{15}{15}$ as $\sim v^{2} \omega_{D}$. This term does contribute to condensation energy and again supports the criterion suggested in Ref $\underline{\underline{10}}$.

\section{DIMENSIONAL ESTIMATES}

Perhaps, the most elementary argument in favor of the discussed criterion can be suggested by applying a 
method of dimensional estimates. Namely, we know that in the limit $\delta \varepsilon \ll \Delta$ the interaction energy is given by Eq. (3). In the opposite limit $\delta \varepsilon \gg \Delta$ it is given again by $v \omega_{D}$, as can be readily obtained by constructing a perturbation expansion in $\mathcal{V}$ around $H_{0}$, i.e., starting from the system of normal electrons (the same result can be easily recovered using Richardson approach). Comparing expressions for the interaction energy in both limits, we immediately arrive at Eq. (10).

\section{DISCUSSION AND SUMMARY}

Thus, a puzzling parameter $\Delta^{2} / \omega_{D}$ turns to be nothing but the binding energy of a single pair. The existence of this quantity in connection to macroscopic results of BCS theory was recently discussed in Refs 16,17 . Actually, based on this quantity, it is possible to develop an interesting interpretation of usual BCS expression (3) for the interaction energy. Standard understanding uses a fact that the interaction between the electrons smears out Fermi energy of noninteracting electrons only within a narrow layer having a width $\sim \Delta$, while the number of such 'excited' pairs is $\sim \Delta / \delta \varepsilon$. By ascribing an energy $\sim \Delta$ to each pair, one recovers Eq. (3) for the interaction energy.

The understanding proposed in Refs $\frac{16,17}{}$ relies on the fact that all electrons within the Debye window are subjected into the interaction, so that the total number of such pairs is $\omega_{D} / \delta \varepsilon$. This is also consistent with the form of the BCS wave function $\underline{\underline{1}}$. The binding energy of each pair is given by a single-pair binding energy $\varepsilon_{c}^{(\text {macro })}$ reduced by the factor of 2 . The reduction is due to the fact that the number of unoccupied electronic states available for the formation of a pair is also twice smaller compared to the Cooper configuration. We thus again recover Eq. (3), but without using an energy scale of $\Delta$. Qualitatively, this understanding is deeply linked to the meanfield concept of each pair being immersed into an average field due to remaining identical pairs. The existence of the energy scale $\varepsilon_{c}^{\text {(macro) }}$ is thus also connected to the validity of the mean-field picture in the macroscopic limit.

The fact that in the many-pair system we still see appearing a single-pair binding energy should be related to the very peculiar form of the BCS reduced Hamiltonian, which contains only coupling between up and down spin electrons on the same energy level, so that pairs feel each other only kinematically, i.e., through the Pauli exclusion principle for the constituent electrons, and not dynamically.

It is easy to realize that although this picture provides new insights to the problem, it does not predict any consequences for measurable quantities in the macroscopic limit at zero temperature, when the mean-field treatment is asymptotically exact. However, as follows from the results of this paper, a hidden energy scale $\varepsilon_{c}^{\text {(macro) }}$, associ- ated with the validity of the mean-field picture, becomes explicit in small-sized systems, when deviations from the mean-field BCS predictions are not negligible anymore. From this viewpoint, the major result of the present paper is rather natural.

Let us stress that the discussed criterion is valid only for those quantities, which are determined by the condensation energy. For those characteristics, which are determined by excitation energies and pair-breaking effects (such as critical temperature $T_{c}$ ), the criterion of Ref ${ }^{\underline{9}}$ must be adequate.

We would like also to mention that, in this paper, we considered the simplest possible model, which enables us to isolate the studied effect from many other important contributions inevitable in real experiments. Among them one can mention level broadening $\frac{19}{9}$, mesoscopic fluctuations 20,21 , and spatially nonuniform pairing 22,23 . The effect of level broadening, however, is more important for temperatures close to $T_{c}$, while we here consider the low-temperature regime ${ }^{19}$. In addition, it is also stronger for smaller systems, in which the mean interlevel spacing is of the order of the superconducting gap. This latter circumstance also applies for mesoscopic fluctuations in the case of disordered grains. For such grains, it was demonstrated ${ }^{20}$ that the crossover between the superconducting state and the fluctuation-dominated regime is smooth, similarly to the case of the equallyspaced model. Therefore, the discussed criterion must be valid for such grains, but one should use a mean interlevel spacing in it. This has to be contrasted with nanosuperconductors of highly-symmetric shapes in a clean limit, for which shell effects as well as a spatially nonuniform pairing take place ${ }^{22,23}$. However, these effects are again more pronounced for smaller systems. Nevertheless, it is also perspective to study in a more detail an accuracy of the mean-field theory for the evaluation of various superconducting characteristics of such systems.

In summary, we discussed a criterion of applicability of the grand-canonical mean-field BCS theory to the evaluation of interaction energy in the ground state of a smallsized system. Based on several approaches and arguments, we demonstrated that the result of this theory for the interaction energy is accurate unless the average difference between the energies of one-electron states becomes of the order of the binding energy of a single Cooper pair. This quantity is much smaller than the superconducting gap.

\section{Acknowledgments}

Useful discussions with M. Combescot are acknowledged. This work was supported by the RFBR (project no. 12-02-00339), RFBR-CNRS programme (project no. 12-02-91055), and Russian Science Support Foundation. 
1 J. Bardeen, L. N. Cooper, and J. R. Schrieffer, Phys. Rev. 108, 1175 (1957).

2 N. N. Bogoliubov, ZhETF 34, 58 (1958) [Sov. Phys. JETP 7, 41 (1958)]; N. N. Bogoliubov, Nuovo Cimento 7, 794 (1958).

3 N. N. Bogoliubov, Physica 26, S1 (1960).

4 J. Bardeen and G. Rickayzen, Phys. Rev. 118, 936 (1960).

${ }^{5}$ D. Mattis and E. Lieb, J. Math. Phys. 2, 602 (1961).

6 O. El Araby and D. Baeriswyl, Phys. Rev. B 89, 134521 (2014).

7 K. Dietrich, H. J. Mang, and J. H. Pradal, Phys. Rev. B 22, 135 (1964); F. Braun and J. von Delft, Phys. Rev. Lett. 81, 4712 (1998).

8 I. L. Kurland, I. L. Aleiner, and B. L. Altshuler, Phys. Rev. B 62, 14886 (2000).

9 K. A. Matveev and A. L. Larkin, Phys. Rev. Lett. 78, 3749 (1997).

10 M. Schechter, Y. Imry, Y. Levinson, and J. von Delft, Phys. Rev. B 63, 214518 (2001).

11 R. W. Richardson, Phys. Lett. 3, 277 (1963).

12 E. A. Yuzbashyan, A. A. Baytin, and B. L. Altshuler, Phys. Rev. B 71, 094505 (2005).

13 L. N. Cooper, Phys. Rev. 104, 1189 (1956).
14 W. V. Pogosov, N. S. Lin, and V. R. Misko, Eur. Phys. J. B 86, 235 (2013).

15 M. Combescot, W. V. Pogosov, and O. Betbeder-Matibet, Physica C 485, 47 (2013).

16 W. V. Pogosov and M. Combescot, Pis'ma v ZhETF 92, 534 (2010) [JETP Letters 92, 534 (2010)]; M. Crouzeix and M. Combescot, Phys. Rev. Lett. 107, 267001 (2011).

17 W. V. Pogosov, J. Phys.: Condens. Matter 24, 075701 (2012).

18 J. Dukelsky, S. Pittel, and G. Sierra, Rev. Mod. Phys. 76, 643 (2004).

19 I. Brihuega, A. M. García-García, P. Ribeiro, M. M. Ugeda, C. H. Michaelis, S. Bose, and K. Kern, Phys. Rev. B 84, 219906 (2011).

20 G. Sierra, J. Dukelsky, G. G. Dussel, J. von Delft, and F. Braun, Phys. Rev. B 61, R11890 (2000).

${ }^{21}$ K. N. Nesterov and Y. Alhassid, Phys. Rev. B 87, 014515 (2013).

22 M. D. Croitoru, A. A. Shanenko, C. C. Kaun, and F. M. Peeters, Phys. Rev. B 83, 214509 (2011).

23 M. D. Croitoru, A. A. Shanenko, F. M. Peeters, and V. M. Axt, Phys. Rev. B 84, 214518 (2011). 